\title{
Indikationsstellung und primäres operatives Vorgehen bei Handinfektionen
}

\author{
Frank Siemers, Philipp A. Bergmann
}

\section{Zusammenfassung}

Weichteilinfektionen im Bereich der Hand sind nicht selten und bedürfen neben einer frühzeitigen Diagnose der schnellstmöglichen Einleitung eines radikalen handchirurgischen Débridements. Im eigenen Patientenkollektiv fanden sich zahlreiche Verläufe mit schwersten Handinfektionen, die häufig auf minimale Verletzungen zurückzuführen waren. Durch z.T. verspätete Vorstellung, wie auch durch eine nicht suffiziente Vorbehandlung, konnten sich rasch fulminante Infekte entwickeln, die nur durch radikale Débridements und lappenplastische Defektdeckungen $\mathrm{zu}$ beherrschen waren. Dauerhafte funktionelle Einschränkungen lassen sich auch bei frühzeitiger Intervention nicht immer verhindern. Eine sofortige adäquate handchirurgische Therapie in einem Zentrum mit entsprechender Expertise kann eine fulminante Infektentwicklung vermeiden.

\section{Indications and Primary Surgical Methods for Hand Infections}

Soft tissue infections of the hand area are not uncommon and require, in addition to an early diagnosis, the initiation of a radical hand surgical debridement as quickly as possible. In our own patient population, numerous courses were found for severe hand infections, that were mainly caused by minimal injuries. Due to the partly belated medical consultation as well as a nonsufficient pretreatment, fulminant infections could rapidly develop which were only treatable through radical debridements and overlapping plastic defects. Permanent functional limitations cannot always be prevented even with an early intervention. Immediate adequate hand-surgical therapy in a centre with appropriate expertise can help to prevent the development of fulminant infections.

\section{Einleitung}

Infektionen an der Hand stellen ein häufiges Krankheitsbild in chirurgischen Abteilungen dar (Abb.1). Die korrekte Diagnose und nicht zuletzt die richtige Therapie von Handinfekten wird nicht selten unterschätzt. Häufig stellen sich Patienten erst nach einem erfolglosen ambulanten Behandlungsversuch in speziellen handchirurgischen Abteilungen vor. Auch Vorbehandlungen in chirurgischen Kliniken ohne handchirurgische Expertise sind keine Seltenheit (Abb. 2 und 3). Dieser Behandlungsversuch besteht meist aus einer alleinigen antibioti-

OP-JOURNAL 2015; 31: 94-98

(C) Georg Thieme Verlag KG Stuttgart · New York DOI http://dx.doi.org/10.1055/s-0035-1545921

schen Therapie oder in einer Kombination mit einer häufig lokal begrenzten chirurgischen Maßnahme wie z.B. einer Punktion oder Stichinzision. Ein erforderliches radikales handchirurgisches Débridement findet nicht immer statt. Nicht selten führen diese frustranen Behandlungsversuche zu nur schwer beherrschbaren Infektsituationen.

\section{Hauptteil}

Bei der Anamnese ist neben der Erfragung eines möglichen Unfallgeschehens und der Berufsanamnese die Erfassung aller relevanter Vorerkrankungen von Bedeutung. In vielen Fällen berichten die Patienten von einer schmerzbedingten Einschränkung der Beweglichkeit. Auch sind nächtliche, oftmals „pochende" Schmerzen mit einem Infektgesche- hen im Bereich der Hand vergesellschaftet.

Neben der Untersuchung im Bereich der Hand ist die Durchführung von Röntgenaufnahmen zur Erfassung röntgendichter Fremdkörper, knöcherner Läsionen und zum Nachweis von Osteolysen obligat. Darüber hinaus sollten laborchemische Daten, die das Vorliegen eines Infektgeschehens sichern (CRP, Procalcitonin, Blutbild) erhoben werden. Bei offenen Wunden, spätestens intraoperativ, erfolgt die Durchführung mikrobiologischer Wundabstriche.

Die Ursache für das Vorliegen eines Handinfekts lässt sich nicht immer sicher ausmachen. Häufig handelt es sich um Mikrotraumata, die initial bagatellisiert werden. Weitere Ursachen sind Einspießverletzungen, Bissverletzungen von Tieren und Menschen (Zahnschlagverletzung) sowie postoperative Wundinfektionen $[4,5,8]$. Eine Sondergruppe, gerade in ländlichen Regionen, stellen Verletzungen bei Personen im landwirtschaftlichen oder fleischverarbeitenden Bereich dar. Bei diesem Patientengut ist die Gefahr der Wundverunreinigung mit MRSA (= Methilicin-resistenten Staphyloccus areus) aufgrund einer hohen Durchseuchungsrate in diesem Kollektiv besonders hoch; fulminante Infektionen werden regelhaft beobachtet. Die am häufigsten nachgewiesenen Keime sind Streptococcus-Spezies, Staphylococcus aureus sowie koagulasenegative Staphylokokken $[3,8]$. Der Nachweis und damit auch die resistenzgerechte antibiotische Therapie bei Infekten an der Hand ist häufig sehr schwierig, da in ca. einem Drittel der Fälle kein Erregernachweis gelingt. Liegt keine sichere Eintrittspforte vor, sind aber deutliche Infektzeichen (Rötung, Schwellung, schmerzbedingte Einschränkung der Beweglichkiet, erhöhte Entzündungsparameter) vorhanden, ist die Indikation für ein operatives Vorgehen großzügig zu stellen. Ursächlich kann immer einmal die Folge einer 


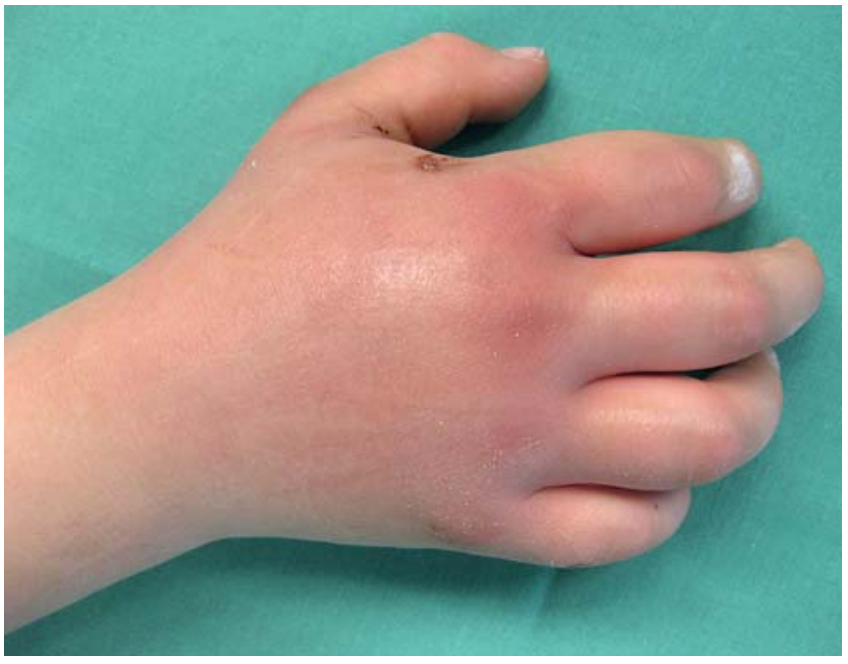

Tab. 1 Klassifikation von Infekten im Bereich der Hand.

\begin{tabular}{ll}
\hline Paronychie & Entzündung im Bereich des Nagelwalls \\
\hline Panaritium subunguale & Entzündung des Nagelbetts \\
\hline Panaritium cutaneum & Eiterblase intradermal \\
\hline Panaritium subcutaneum & subkutaner Infektherd \\
\hline Panaritium tendinosum & Entzündungsprozess innerhalb der Sehnenscheide \\
\hline Panaritium ossale & Osteitis oder Osteomyelitis \\
\hline Panaritium articulare & Gelenkempyem
\end{tabular}

hämatogenen Einschwemmung im Handbereich vorliegen.

Infekte im Bereich der Hand werden nach ihrer Lokalisation und Ausdehnung unterteilt (Tab. 1). Ist bei pyogenen Entzündungen der Nagelwall betroffen, handelt es sich um eine Paronychie, Panaritien betreffen das übrige Gewebe.
Abb. 1 Infekt Hand-

rücken. dermale Entzündungsprozesse (Panaritium cutaneum) von subkutanen Infekten (Panaritium subcutaneum). Ist das Sehnengleitgewebe betroffen, kann sich das Infektgeschehen leicht über den präformierten Raum vom Finger bis in die Hohlhand ausbreiten, in diesem Fall liegt dann ein Panaritium tendinosum vor. Einen Sonderfall des Panaritium tendi- nosum stellt die V-Phlegmone dar. Hierbei kommt es durch die kommunizierenden Beugesehnenscheiden des 1. und 5. Strahles zu einem Übergang des Infektgeschehens auf beide Strahlen. Liegen entzündliche Veränderungen intraartikulär vor, wie es nicht selten bei Einstichverletzungen, aber auch Zahnschlagverletzungen der Fall ist, liegt ein Panaritium articulare vor. Panaritium ossale beschreibt entzündliche Veränderungen intraossär.

Bei fulminanten Infekten ist eine Infektbeherrschung nur durch ein radikales chirurgisches Débridement zu erreichen. Die Grundlage dieses radikalen chirurgischen Débridements an der Hand stellt fundierte Grundkenntnis über die komplexe Anatomie der Hand und die dortigen funktionellen Strukturen dar [1] (Abb. 4 und 5).

In der eigenen Klinik favorisieren wir beim Vorliegen entzündlicher Veränderungen im Bereich der Hand ein mehrzeitiges Vorgehen. Beim radikalen Débridement wird ausgehend von einer nachweislich vorhandenen Eintrittsstelle eine Inzision mit Schnitterweiterung vorgenommen. Lässt sich eine Eintrittspforte nicht sicher identifizieren, erfolgt die Inzision in der Region mit der ausgeprägtesten Rötung und Schwellung.

Der operative Eingriff erfolgt in Rückenlage des Patienten bei einem auf einem Armtisch ausgelagerten Arm in Oberarmblutsperre. Auf die Anlage einer Blutleere wird aufgrund der Gefahr der Keimverschleppung verzichtet. In der Regel wird eine Allgemeinnarkose durchgeführt. Nur bei lokal begrenztem Infekt-

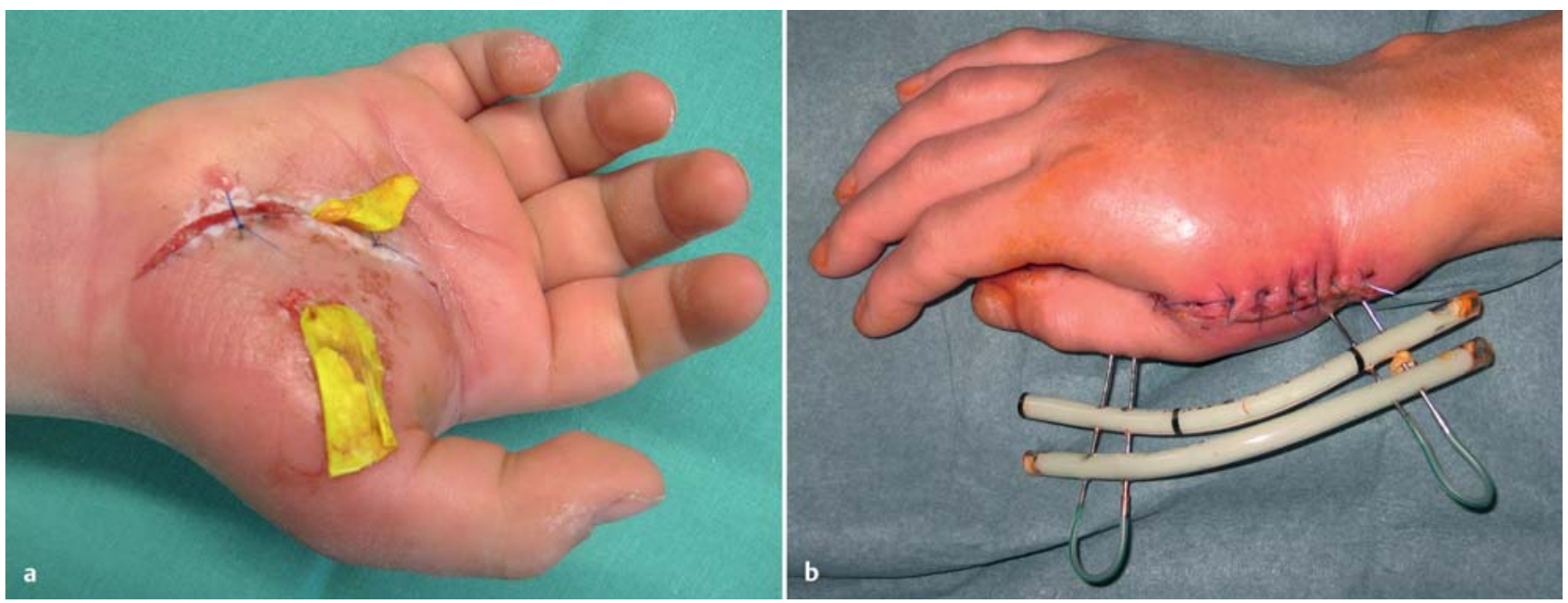

Abb. 2 a und b Aufnahmebefunde nach auswärtiger operativer Vorversorgung bei lokalen Infekten im Bereich der Hand.

Frank Siemers, Philipp A. Bergmann: Indikationsstellung und primäres operatives Vorgehen bei Handinfektionen 


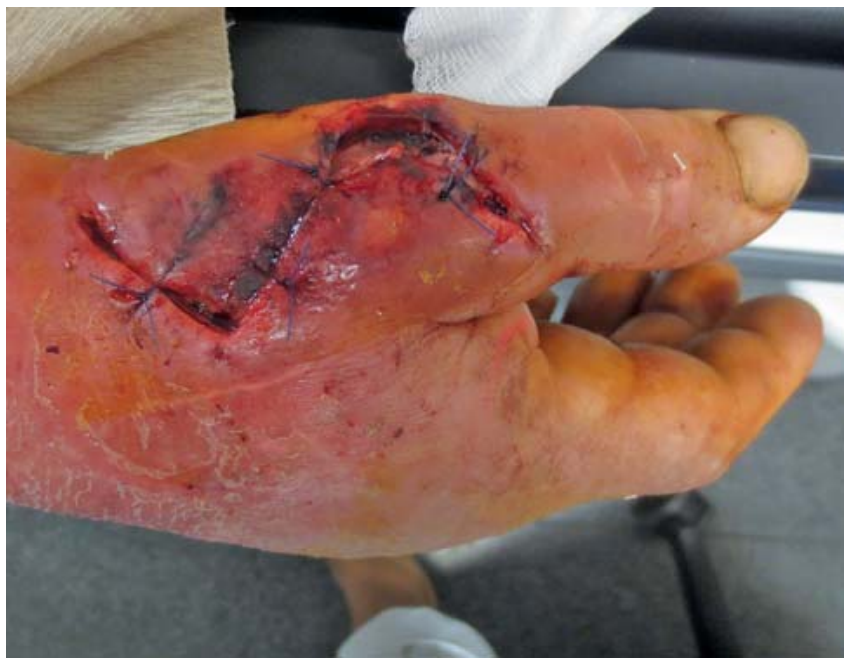

Abb. 3 Streckseitiger Infekt über dem 1. Strahl bei auswärtiger Erstversorgung.

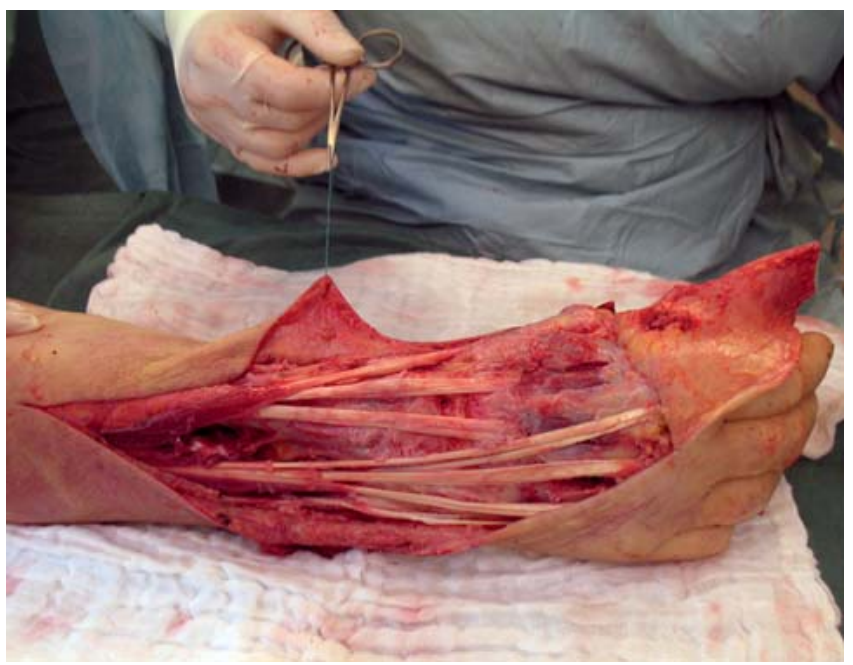

Abb.5 Befund nach Débridement mit freiliegenden Strecksehnen und Haut-Weichteil-Defekt.

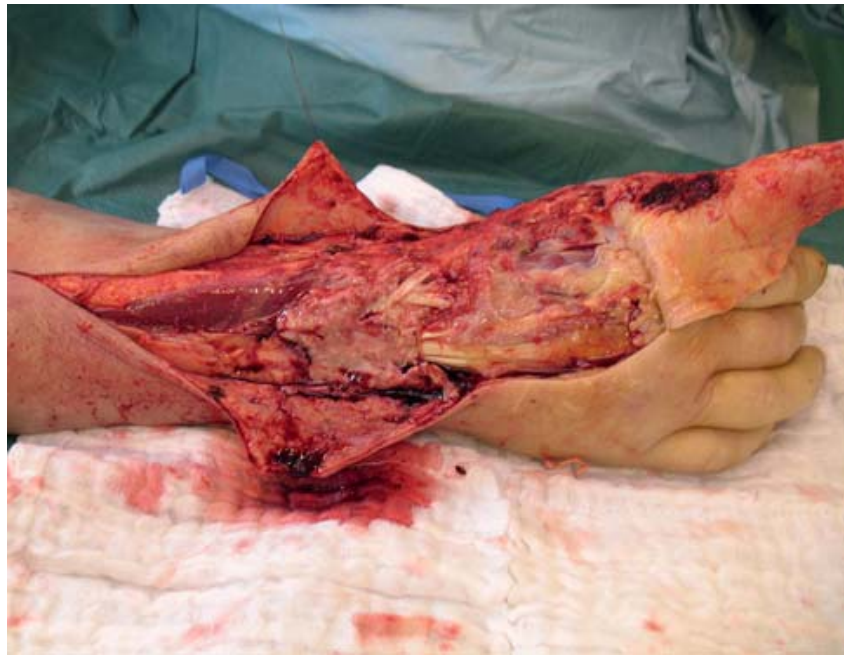

Abb. 4 Intraoperativer Befund vor Débridement.

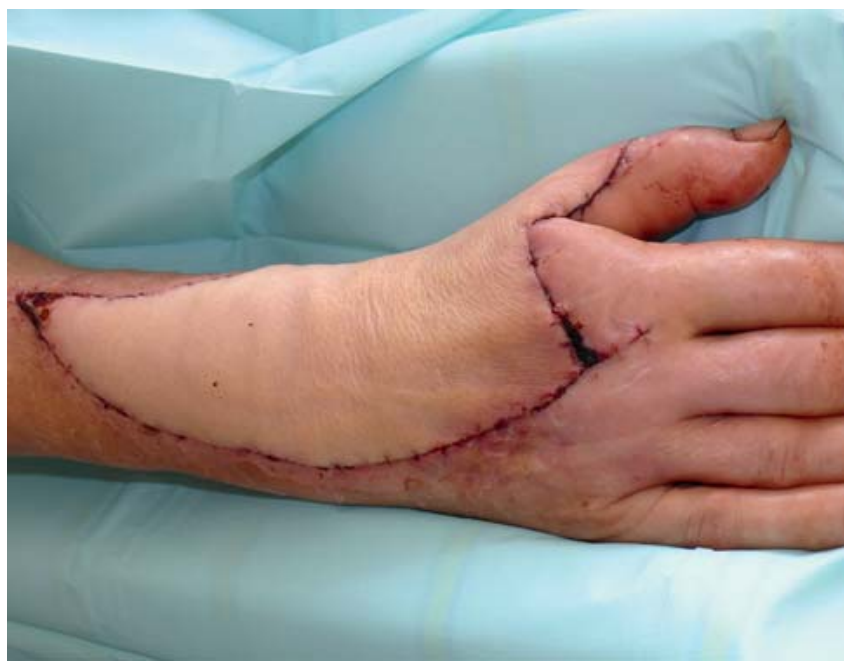

Abb. 6 Plastisch-chirurgische Defektdeckung mittels freier ALT-Lappenplastik (freier Oberschenkellappen). geschehen, bei dem das Vorliegen einer Lymphangitis sicher ausgeschlossen ist, kann auch eine Plexusanästhesie durchgeführt werden.

Bei der OP-Planung werden die klassischen handchirurgischen Schnittführungen berücksichtigt. Palmar sind dies zickzackförmige Schnitte nach Bruner oder Inzisionen mediolateral (Abb.2b) Streckseitig kommen bevorzugt bogenförmig geschwungene Hautinzisionen zum Einsatz. Bei fortgeschrittenen Infektsituationen kann es erforderlich sein, kompromittierte Hautbereiche $\mathrm{zu}$ entfernen. Hieraus resultieren dann lappenpflichtige Defekte, die nach erfolgreicher Sanierung des Infektionsherds plastischchirurgisch gemäß der rekonstruktiven Leiter zu decken sind (Abb. 6).
Im Rahmen des Débridements werden alle sichtbar entzündlichen Veränderungen operativ entfernt. Die Gefäß-NervenBündel werden im Rahmen der Operation dargestellt und geschont. Im Umfeld von entzündlichen Prozessen ist die Abgrenzung der funktionellen Strukturen anspruchsvoll, die Nutzung einer Lupenbrille sollte unbedingt erfolgen.

Das chirurgische Konzept wird von einer perioperativen intravenösen Antibiotikatherapie mit Cefuroxim 1,5 g flankiert [2]. Patienten mit bekannter Allergie erhalten Ciprofloxacin $400 \mathrm{mg}$ intravenös. Nach Abschluss des radikalem Débridements nach den Regeln der Handchirurgie folgt eine Wundspülung mit anschließender Anlage eines PolyhexanidgelFettgaze-Verbands, der zusätzlich für die nachfolgenden 12 Stunden in einem 2-stündlichen Intervall von außen mit Polyhexanidlösung befeuchtet wird. Die Wunde wird an den BG-Kliniken Bergmannstrost offen behandelt. Zahlreiche Kliniken legen adaptierende Wundnähte vor. Eine begleitende Schienenruhigstellung mit Hochlagerung wird bei allen Patienten durchgeführt. Aufgrund des Feuchtverbands empfiehlt sich die Verwendung von Kunststoffschienen.

Bei Infekten, die nicht lokal begrenzt waren, wird die intraoperativ begonnene Antibiotikatherapie für mindestens 5 weitere Tage fortgesetzt. Die intravenöse Therapie mit dem Antibiotikum $(3 \times 1,5 \mathrm{~g})$ kann bei Stabilisierung des klinischen Bildes auf eine orale Therapie umgestellt werden $(2 \times 500 \mathrm{mg}$ per os) Nach Keimisolierung und Antibiogramm 
wird die antibiotische Therapie, wenn nötig, resistenzgerecht umgestellt.

Am 1. postoperativen Tag erfolgt im Rahmen der Visite die Re-Evaluation der lokalen Situation. War das initiale Débridement einmal nicht ausreichend, kann der zu diesem Zeitpunkt noch nüchterne Patient einem nochmaligen operativen Débridement zugeführt werden. In den allermeisten Fällen hat sich die Weichteilsituation soweit stabilisiert, dass der sekundäre Wundverschluss für den Folgetag geplant werden kann. Falls erforderlich, kommen bei diesem Zweiteingriff auch plastisch-rekonstruktive Verfahren wie Spalthauttransplantationen oder Lappenplastiken zum Einsatz. Während des stationären Aufenthalts wird frühzeitig mit handrehabilitativen Maßnahmen begonnen. Neben der Physiotherapie hat die manuelle Lymphdrainage einen sehr hohen Stellenwert.

In einer aktuellen retrospektiven Analyse haben wir die Notwendigkeit einer zeitnahen und radikalen chirurgischen Therapie bei schwersten Infektionen im Bereich der Hand herausgearbeitet. Für das Jahr 2013 konnten 13 Patienten (9 männliche und 4 weibliche) identifiziert werden, bei denen folgende Kriterien zutrafen:

1. Mehr als 3 notwendige chirurgische Débridements zur Infektsanierung.

2. Ein stationärer Aufenthalt, der aufgrund des Handinfekts deutlich über dem Durchschnitt von 4,9 Tagen liegt.

3. Die Notwendigkeit zur lokalen oder freien Lappenplastik im Verlauf zur Defektdeckung.

Alle Betroffenen wurden nach durchschnittlich 7,4 Monaten (6,1-12,3) nachuntersucht. Neben dem DASH-Score wurde die Beweglichkeit im Bereich der oberen Extremität erhoben. Eine Handkraftmessung sowie eine Fotodokumentation schlossen sich an.

Die 13 Patienten hatten ein Durchschnittsalter von 58,8 (43-76) Jahren. Sechs dieser Patienten stellten sich initial in unserer Klinik vor, 7 Patienten wurden auswärts vorbehandelt. Durchschnittlich 8,34 Tage nach Unfall wurden die Patienten in unserer Klinik vorstellig. Die Gruppe der Erstvorstellungen bei uns stellte sich nach durchschnittlich 3,83 Tagen, die vorbehandelten Patienten nach durchschnittlich 12,29 Tagen vor. Sieben Patienten stellten sich mit ausgeprägten Phlegmonen der gesamten Hand, die partiell auf den Unterarm rei- chend waren, vor, bei 3 Patienten lagen Beugesehnenscheidenphlegmonen vor, 2 davon jedoch im Übergang in eine ausgeprägte Handphlegmone. Drei Patienten präsentierten eine auf den Daumen begrenzte Infektion im Sinne eines Panaritium subcutaneum, ein weiterer Patient stellte sich mit einer postoperativen Wundinfektion vor, die zu einer ausgeprägte Phlegmone des Mittelfingers führte.

$\mathrm{Zu}$ beschriebenen Handinfekten führten in 6 Fällen ein Bagatelltrauma, in 3 Fällen ein postoperativer Wundinfekt und in je 2 Fällen Katzenbisse und Schnittverletzungen im fleischverarbeitenden Betrieb.

Bei beiden letztgenannten Patienten konnte MRSA in der Wunde festgestellt werden, übrige Keime waren in 4 Fällen Streptococcus-Sspezies, in 4 Fällen Staphylococcus aureus und je in 1 Fall Klebsiellen und Pasteurella multocida. In 1 Fall konnte kein Keim nachgewiesen werden. Bei 4 der Patienten war in der Vorgeschichte ein Diabetes mellitus bekannt, 3 Patienten waren Raucher.

Die Vorbehandlung der 7 Patienten war in 2 Fällen auf eine alleinige Gabe eines Antibiotikums beschränkt, in 5 Fällen fand eine zusätzliche chirurgische Therapie in Form einer Stichinzision statt. In 1 Fall wurde ein ausgedehnteres Débridement durchgeführt. Die jeweiligen chirurgischen Therapien fanden alle in nicht handchirurgischen Zentren statt.

Die durchschnittliche stationäre Verweildauer lag bei 26,77 (11-37) Tagen, im Durchschnitt waren 2,69 Débridements notwendig, um einen sekundären Wundverschluss durchzuführen. Bei dem von uns untersuchten Kollektiv gelang der direkte sekundäre Wundverschluss nur in 1 Fall, in 1 Fall musste der betroffene Finger amputiert werden, in 2 Fällen erfolgte die Defektdeckung durch eine DMCA-Lappenplastik (DMCA: Distal-metacarpophalangeal-Arterien), in 4 Fällen durch einen Bauchhautlappen sowie in 5 Fällen durch eine freie ALTLappenplastik (ALT: anterolateral thigh).

Nach sekundärem Wundverschluss bzw. Defektdeckung durch lokale oder freie Lappenplastiken kam es zu einer reizlosen Wundheilung, keine Wunde musste revidiert werden. Die stationären physiotherapeutischen Beübungen konnten nach Wundstabilisierung begonnen werden.
Eine durchschnittliche Minderung des DASH-Scores nach 6 Monaten lag bei 37 (5-68,3) Punkten, wobei der durchschnittliche postoperative DASH-Score 48 (5-68,3) Punkte aufweist. Elf Patienten zeigten postoperativ eine erhebliche Einschränkung in der Beweglichkeit des Handgelenks sowie der Langfinger. Die durchschnittliche Minderung der Handgelenksbeweglichkeit beträgt $23 \%$, die der Langfinger 30\% in Bezug auf die gesunde Gegenseite. Im Vergleich zur Gegenseite ergibt sich eine Minderung der Grobkraft von 37\%, sowie eine Minderung der Feinkraft von 31\%.

Das nachuntersuchte Kollektiv präsentiert eine Zusammenschau an schweren Handinfekten, die aus unterschiedlichen Gründen zu einer Infektexazerbation führten und eine ausgedehnte handund plastisch-chirurgische Therapie zur Infektsanierung nach sich zogen. Gründe hierfür waren in der Mehrzahl eine verspätete Vorstellung mit einer nicht ausreichenden Vorbehandlung sowie das Vorliegenden von zusätzlichen Risikofaktoren. Die richtige Therapie und die zeitnahe Indikation zur chirurgischen Behandlung bei Handinfekten sind bei mangelnder Erfahrung häufig erschwert und werden unterschätzt.

In der Literatur wird eine Vielzahl an Empfehlungen zur Therapie von Handinfekten beschrieben, wobei die Wahl der Methode abhängig von der Erfahrung und Ausbildung des Operateurs ist. Konsens besteht bez. eines möglichst frühzeitigen und radikalen chirurgischen Débridements. Die rasche Diagnosestellung und anschließende zeitnahe und adäquate Therapie ist entscheidend für das Outcome und erhöht die Chancen auf eine vollständige Rehabilitation [6]. Das vorgestellte 2-zeitige Therapieregime stellt eine sichere Option dar. Für den Fall, dass das initale Débridement einmal nicht ausreichend war, kann frühzeitig die Re-Intervention erfolgen. Der definitive Wundverschluss erfolgt erst, wenn das Infektgeschehen beherrscht ist.

Der Alltag in chirurgischen Kliniken in Deutschland zeigt, dass in Bezug auf die Behandlung von Handinfekten große Unterschiede in der Therapie und Behandlungskonsequenz bestehen. Ausmaß und Folgen einer Infektion im Bereich der Hand werden nicht selten unterschätzt. Die Folge sind nicht ausreichende Maßnahmen. Ambulante Behandlungen mit konservativen Therapieversuchen mittels Salbenverbänden und 
Antibiotikabehandlungen sind nicht die Ausnahme und so werden immer noch viele Patienten nicht frühzeitig genug einem radikalen handchirurgischen Débridement zugeführt. Auch der ökonomische Faktor wird häufig unterschätzt: Reichert et al. [7] konnten nachweisen, dass aufgrund initialer Fehleinschätzung und Fehlbehandlung von Handinfekten insgesamt erhebliche Mehrkosten entstanden sind. Ziel sollte also sein, Patienten, die eine floride Infektion oder auch den Verdacht einer floriden Infektion an der Hand zeigen, in ein handchirurgisches Zentrum zu verlegen bzw. direkt dort vorzustellen, um so eine vollständige Behandlung zu gewährleisten.

\section{Schlussfolgerung}

Infekte im Bereich der Hand können bei initial vermeintlich harmloser Entzündungssituation schnell in einen exazerbierten Zustand übergehen. Das Bewusstsein für eine solche mögliche Ent- wicklung muss bei allen Behandelnden geschärft werden. Nur durch eine frühzeitige Behandlung in einer Einrichtung mit ausgewiesener handchirurgischer Kompetenz können zeitnah die erforderlichen Entscheidungen getroffen werden und entsprechende operative Maßnahmen in die Wege geleitet werden.

\section{Literatur}

${ }^{1}$ Bergmann PA, Mauss KL, Liodaki E et al. Handinfektionen: Evaluation eines zweizeitigen Therapieregimes. Handchir Mikrochir Plast Chir 2014; 46: 49-55

2 Bodmann K-L, Grabein B, e.V. EdP-E-GfC. Empfehlungen zur kalkulierten parenteralen Initialtherapie bakterieller Erkrankungen bei Erwachsenen, Update 2010. Chemother J 2010; 19: 179-255

${ }^{3}$ Houshian S, Seyedipour S, Wedderkopp N. Epidemiology of bacterial hand infections. Int J Infect Dis 2006; 10: 315-319

${ }^{4}$ Kall S, Vogt PM. Chirurgische Therapie von Infektionen der Hand I. Chirurg 2005; 76: 615625

5 Kall S, Vogt PM. Chirurgische Therapie von Infektionen der Hand II. Chirurg 2005; 76: 721730
${ }^{6}$ Reh-Plass S, Schaller E. Stellenwert der Frühintervention bei Handinfektionen. Handchir Mikrochir Plast Chir 1991; 23: 214-217

7 Reichert B, Zöphel O, Möller $M$ et al. Behandlungskosten durch unterschätzte Handinfektionen. Handchir Mikrochir Plast Chir 2001; 33: 354-358

${ }^{8}$ Weinzweig N, Gonzalez M. Surgical infections of the hand and upper extremity: a county hospital experience. Ann Plast Surg 2002. 49: 621-627

\section{PD Dr. med. Frank Siemers}

Direktor der Klinik für Plastische und Handchirurgie/Brandverletztenzentrum

\section{Dr. med. Philipp A. Bergmann}

Assistenzarzt

Klinik für Plastische und Handchirurgie/Brandverletztenzentrum Berufsgenossenschaftliche Kliniken Bergmannstrost Merseburger Straße 165 06112 Halle (Saale)

frank.siemers@bergmannstrost.com 